\title{
"RadioAstron" Mission Correlator Features and Operations
}

\section{V.I. Kostenko', A.S. Andrianov, V.Yu. Avdeyev, K.G. Belousov,}

\section{I.A. Guirin, S.F. Likhachev, M.V. Shatskaya}

Astro Space Center of Lebedev Physical Institute,

Moscow, Russian Federation

E-mail:vkostenko@asc.rssi.ru

\section{V.E. Zharov}

Sternberg Astronomical Institute

Moscow, Russian Federation

E-mail:: vladzh2002@yandex.ru

The 'Radioastron' space mission is the unique project of Russian Space Agency (Roscosmos) and Russian Academy of Sciences to investigate the Universe by means of implementation of VLBI principles with 'Spectr-R' space vehicle.

The 10-m radiotelescope onboard 'Spectr- $\mathrm{R}$ ' has been fully operational since 15 November, 2011 as the Space element of Ground-to-Space interferometer at the orbit with the apogee up to $350000 \mathrm{~km}$. The correlator for Radioastron mission is a part of ASL (Astro Space Locator) computer complex for Windows environment fully developed at Astro Space Center (ASC). In the report the main features and operational procedures of ASL correlator are described. Methods of extracting of the correlation results are analyzed in terms of efficiency and parameterization of correlator. The scientific goals are clearly illustrated by a number of Radioastron Early Science Program observing sessions.

11th European VLBI Network Symposium \& Users Meeting

October 9-12, 2012

Bordeaux, France

\footnotetext{
${ }^{1}$ First author
} 


\section{Introduction}

On July 18, 2011 the Astro Space Center (ASC) of P.N.Lebedev of Physical Institute of the Russian academy of sciences and Federal Space Agency of Russian Federation in collaboration with many world known scientific organizations accomplished successful launch on Earth orbit of the unique radioastronomy observatory "Radioastron" with $10 \mathrm{~m}$ precision reflector antenna onboard. As for today "Radioastron" successfully works for more than one year, jointly with the largest Earth based radiotelescops and so forms a huge multi element interferometer with maximum baseline up to $350000 \mathrm{~km}$ and angular resolution down to some micro seconds of arc. Table 1 illustrates current in flight specifications of Radioastron mission [1].

\begin{tabular}{|l|r|r||r|r|}
\hline \multicolumn{1}{|c|}{ Parameter } & $\lambda=1.35 \mathrm{~cm}$ & $\lambda=6.2 \mathrm{~cm}$ & $\lambda=18 \mathrm{~cm}$ & $\lambda=92 \mathrm{~cm}$ \\
\hline Ө0.5 $\pm 5 \%$, arc $\min$ & $6 \times 13$ & 25 & 72 & 60.1 \\
\hline Aeff $\pm 10 \%, \mathrm{~m} 2$ & 7.5 & 35 & 42 & 30 \\
\hline Tsys $\pm 10 \%, \mathrm{~K}$ & 77 & 130 & 45 & 200 \\
\hline SEFD $\pm 10 \%, \mathrm{Jy}$ & 30000 & 10250 & 3000 & 18400 \\
\hline$\Delta v \mathrm{IF}, \mathrm{MHz}$ & \pm 16 & \pm 16 & \pm 16 & 4 \\
\hline $\begin{array}{l}12 \sigma \mathrm{Ra}-\mathrm{GBT}, 300 \\
\text { Sec, } \mathrm{mJy}\end{array}$ & 240 & 80 & 50 & 480 \\
\hline $\begin{array}{l}\text { Acorr } \\
(12 \sigma \text { Ra-GBT })\end{array}$ & $2.3 \mathrm{e}-4$ & $1.8 \mathrm{e}-4$ & & - \\
\hline
\end{tabular}

Table 1. "Radioastron" in flight (measured) specifications.

\section{Description of Astro Space Center correlator and its functionality}

ASC correlator is a major element of space interferometer. Its outputs serve as aggregate quality measure of whole Radioastron mission and mainly determine the success of intended scientific goals. The correlator itself is an important engine of ASC Scientific Data Processing Center (SDPC). Main external data (from Tracking station) and communication channels (commHost, Internet cloud) along with internal exchange functional channels are shown in Figure 1. The main operational features of ASC Software FX-mode Correllator are shown in Figure 2.

ASC FX Correlator comprises well recognized and useful functions needed for everyday correlation. It is ASL computer application package for Windows environment fully developed at Astro Space Center (ASC) [2]. The ASL software correlator main features are following:

- RDF (Russian design) - Mark5(*), K5 formats universal data input.

- More than 1 Tflops / sec Cluster efficiency (estimated);

- 200 TB disk storage supported;

- $256 \mathrm{Mb} / \mathrm{sec} / \mathrm{station}$ data flow, 10 stations (45 base lines) tested; 
- More than 65536 delay channels (tested);

- Times 2 efficiency of real time processing (4-stations, 6 BL's), see Figure 3;

- Continuum, Line and Pulsar operational modes;

- User friendly execution management and smart GUI.

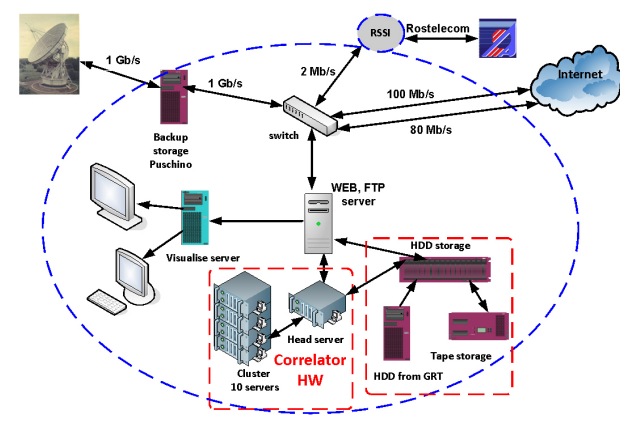

Figure 1. ASC Scientific Data Processing Center infrastructure. Data acquisition, transfer and processing

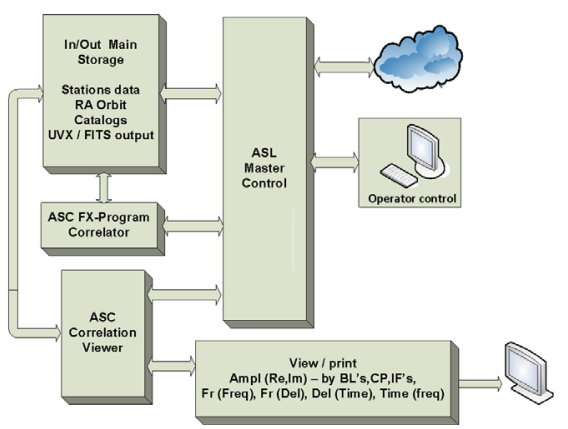

Figure 2. ASC Software Correlator environment. FX mode.

Correlator output can be easily, elegantly analyzed and visualized by ASL user applications library with a variety of important modes of operation:

- Flexible analysis at Fr-Del-Freq-Time space;

- Data collection, sorting \& discarding, calibration and interactive image restoration;

- 1-2-3 dimensional screen views \& plots (graphs \& tables).

ASL applications are compatible with FITS-IDI, UVFITS formats and existing post processing applications (AIPS, PIMA etc.).

ASC Correlator efficiency

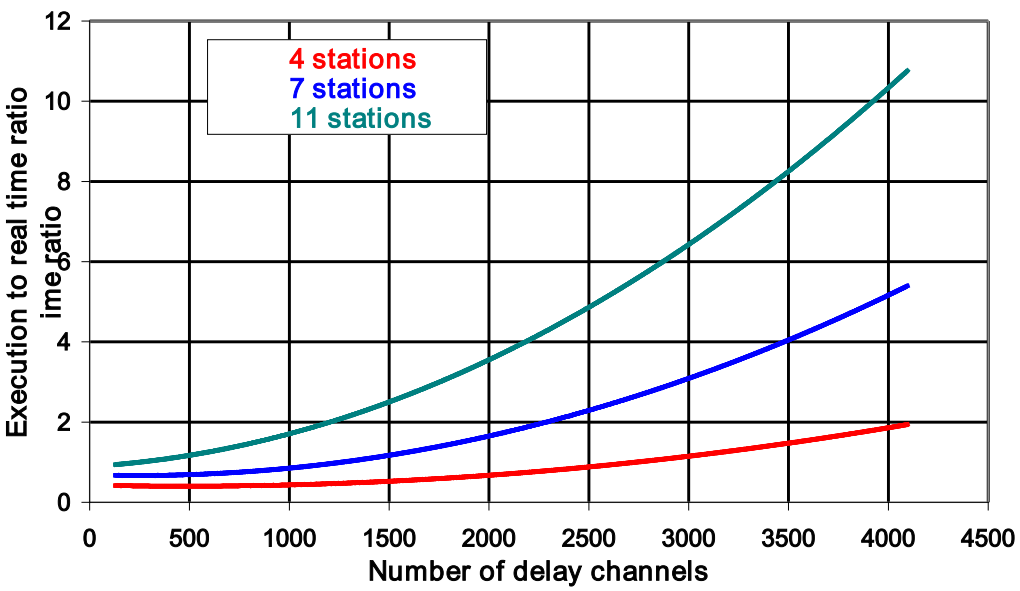

Figure 3. ASC correlator 'real time efficiency-number of channels' relationship

Correlation results strictly depend on the approximated delay model of the interferometer. Uncertainties of "RA" reconstructed orbit put evident restrictions to the trial Fringe search procedure. Chancy combination of integration parameters, including delay, velocity and 
RA accelaration Fringe Search window $\mathrm{dV} / \mathrm{dt}\left(\mathrm{mm} / \mathrm{sec}^{2}\right)$

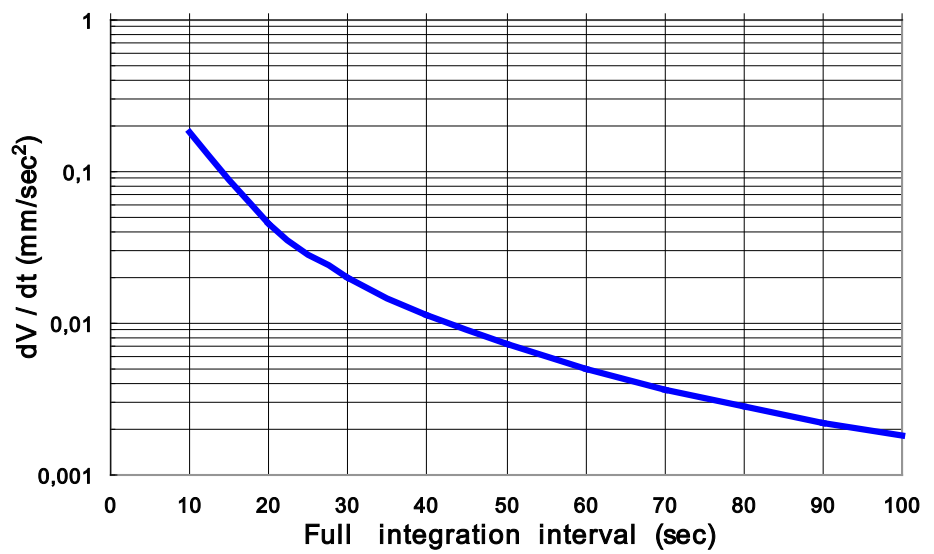

Figure 4. RA acceleration (dv/dt) set limit to the integration interval.

acceleration of RA observatory, should be accurately adjusted to identify correlation response with the adequate sensitivity. Accordingly fringe search window is determined by RA state vector, velocity and acceleration prediction. Those values are critical for right choice of the number of spectral channels and partial/total integration intervals. The corresponding relationship is illustrated in Figure 4.

The search procedure to Maximize correlated signal to noise ratio in a range of $\mathrm{dv} / \mathrm{dt}$ will help to rise probability of signal extraction up to the level of natural coherence time. The method has been used by ASC FX correlator and ASC diFx group and shows evident efficiency for both. An example of such approach is shown in Figure 5.

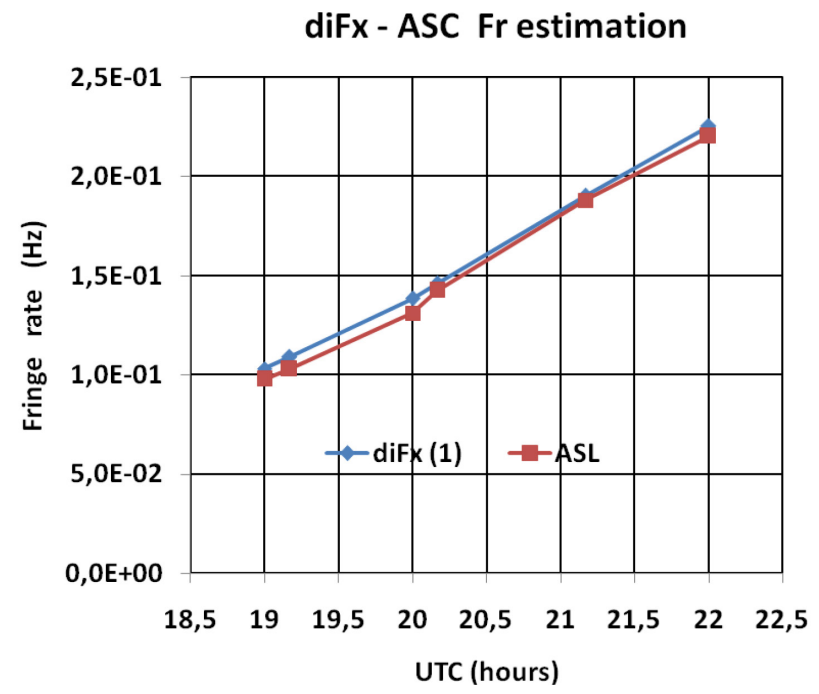

Figure 5. Comparison of ASC and diFx results shows closeness of 'fringe frequency' estimation by different applications. 


\section{Processing statistic and correlation examples}

After one year of Radioastron project active life two investigation programs are taking place: Fringe search program (November 2011- February 2012) and Early science program (February 2012 - continue) [3].

For today a number of radio sources of different classes have been observed. Figure 6 demonstrates observing session's quantitative relations by source categories, processing stages and results.

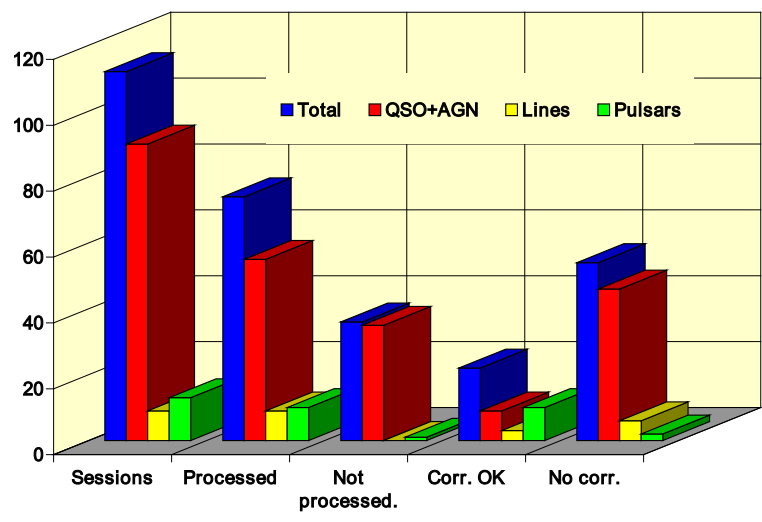

Figure 6. Radioastron mission data processing status, February-August, 2012. Number of events versus source categories, processing stages and correlation results.

In conclusion the following figures depict two pronounced correlation results of continuum and line processing modes.
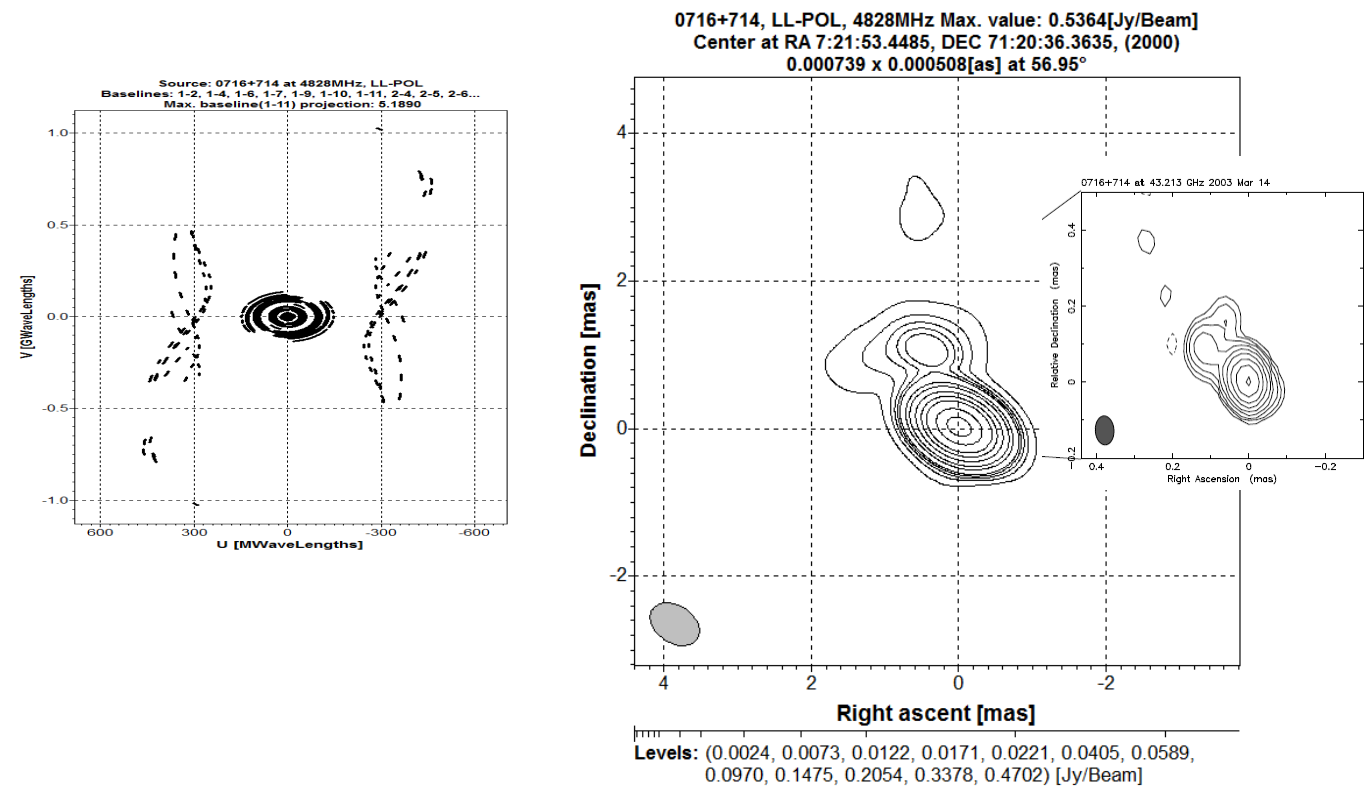

Figure 7. Radioastron- Earth coverage (left insert) and trial image (right insert) of 0718+714 source at $4828 \mathrm{MHz}$. Stations are RA, EF, BD, ZC, YS, MC, WB, UR, SH. Compare $0718+714$ image at $43 \mathrm{GHz}$ (far right insert, Uwe Bach. Dissertation, Cologne, 2004). 
Figure 7 demonstrates Space VLBI trial image (Sergey Likhachev, ASC) of the BL Lacertae object 0716+714 reconstructed from data of RadioAstron-EVN 24 hours session at 6.2 $\mathrm{cm}$. The image is reconstructed with resolution of $0.74 \times 0.51$ mas. The peak brightness is 0.47 Jy/beam.
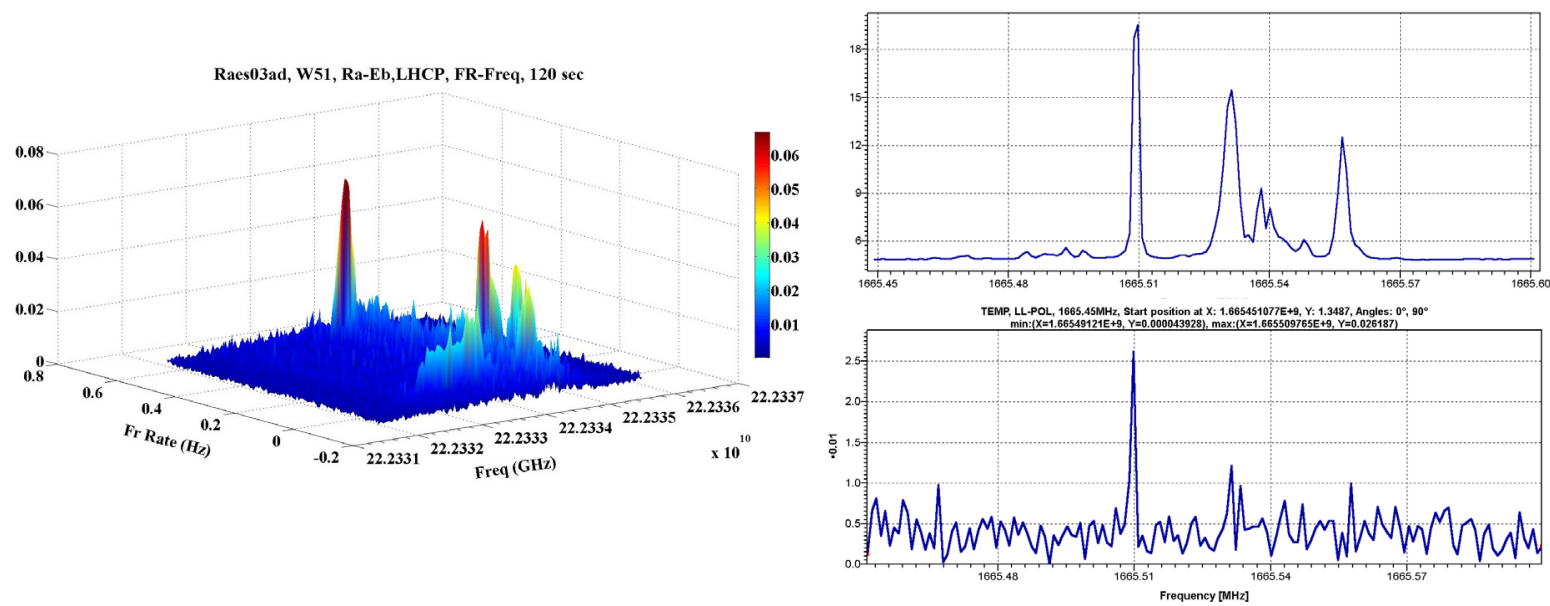

Figure 8. W51 H2O spectral line correlation results at Radioastron-Effelsberg baseline.

Figure 8 shows processing results for the $\mathrm{H}_{2} \mathrm{O}$ maser line complex in the W51 star forming region viewed as sequence of plots from autocorrelation (Effelsberg, right-top) to cross correlation (Radioastron-Effelsberg, right-bottom) and 'fringe rate-frequency' 3-D plot (left). Observing date is May 12, 2012, projected baseline 1.14 of Earth diameters and integration time 120 seconds.

\section{References}

[1] F. V.Yu. Avdeyev, A.V.Alakoz, Yu.A. Andreyanov, et al., "Radioastron" space mission. First results, Vestnik, NPO S.A. Lavochkin association Science \& technical journal, Russian edition, №3, p.4, 2012.

[2] M.V. Shatskaya, A.A. Andrianov, I.A. Guirin et al., Set-up of Scientific information Processing Center for radiointerferometry projects, Kosmicheskiye issledovaniya, v 50, №4, pp. 346-350, 2012.

[3] Radioastron Newsletter \# 4, 10-12, www.asc.rssi.ru/radioastron/news/newsl/en 